\title{
Olfactory Sex Discrimination Persists, Whereas the Preference for Urinary Odorants from Estrous Females Disappears in Male Mice after Vomeronasal Organ Removal
}

\author{
Diana E. Pankevich, ${ }^{1}$ Michael J. Baum, ${ }^{1}$ and James A. Cherry ${ }^{2}$ \\ Departments of ${ }^{1}$ Biology and ${ }^{2}$ Psychology, Boston University, Boston, Massachusetts 02215
}

\begin{abstract}
Based on observed changes in the social context for the display of ultrasonic vocalizations, scent marking, aggression, and mounting behavior by male mice with a null mutation of the transient receptor potential 2 ion channel, it was proposed recently that a primary function of the mouse vomeronasal organ (VNO)/accessory olfactory system is sex discrimination. We tested this hypothesis directly by studying the ability of male mice to discriminate between urinary odors of conspecifics of the two sexes and in different endocrine states using habituation- dishabituation tests. Male mice from which the VNO had been surgically removed (VNOx) resembled sham-operated controls (VNOi) in their ability to discriminate between volatile urinary odors from estrous females versus gonadally intact males, as well as between urinary odors from estrous versus ovariectomized females and from gonadally intact versus castrated males. When physical access to stimuli was permitted, VNOi control males strongly preferred to investigate volatile and nonvolatile urinary odorants from estrous females as opposed to intact males, whereas VNOx males showed no such preference. Mating performance in tests with estrous females was equivalent in VNOi and VNOx subjects. Both groups of males preferred to mount an estrous female instead of a castrated male. Our results suggest that the $\mathrm{VNO}$ is not required for sex discrimination but instead detects the nonvolatile components of oppositesex urine that may be used to help prolong contact with individuals that produce these chemosignals.
\end{abstract}

Key words: sexual behavior; pheromone; accessory olfactory bulb; odor detection; habituation; urinary

\section{Introduction}

Aspects of social communication in male mice depend in part on the activation of receptor neurons in the vomeronasal organ (VNO) by nonvolatile components of urine or other body odorants (Halpern and Martinez-Marcos, 2003). Recently, the VNO has been genetically manipulated in male mice by mutating a cluster of VNO receptor genes (V1r) (Del Punta et al., 2002), the related $\mathrm{G}_{\alpha 2 \mathrm{i}}$-protein (Norlin et al., 2003), the $\mathrm{p} 73$ gene (Yang et al., 2000), or the transient receptor potential 2 (TRP2) cation channel (Leypold et al., 2002; Stowers et al., 2002). Null mutations of TRP2 $\left(T_{R P 2}{ }^{-/-}\right)$resulted in a loss of in vitro multiunit responses in VNO neurons and in a reduction of VNO local field potentials measured after exposure to urinary odorants. TRP2 $2^{-/-}$males also showed high levels of mounting behavior and reduced levels of aggression toward an intruder male (Leypold et al., 2002; Stowers et al., 2002). It was concluded that the VNO is required for sex discrimination (Stowers et al., 2002) and for the detection of odorants that determine sex partner preference (Leypold et al., 2002).

The claims made in these two studies that deletion of the TRP2

Received March 29, 2004; revised Aug. 26, 2004; accepted Sept. 7, 2004.

This work was supported by National Institutes of Health Grant MH59200. We thank Erin Deedy and Kristine Martel for technical assistance.

Correspondence should be addressed to Dr. James Cherry, Department of Psychology, Boston University, 64 Cummington Street, Boston, MA 02215. E-mail: jcherry@bu.edu.

DOI:10.1523/JNEUROSCI.2376-04.2004

Copyright $\odot 2004$ Society for Neuroscience $\quad$ 0270-6474/04/249451-07\$15.00/0 channel eliminated the ability of male mice to discriminate the sex of conspecifics were based primarily on the observation that $T R P 2^{-/-}$males mounted male and female subjects indiscriminately, yet neither paper directly measured olfactory sex discrimination. As pointed out previously (McCarthy and Auger, 2002), it remains questionable whether $T R P 2^{-/-}$males can discriminate between the sexes on the basis of olfactory cues. Nevertheless, the high profile given to these reports on the TRP2 ${ }^{-/-}$phenotype may have led to a widespread (mis)perception that male mice cannot distinguish males from females without the processing of pheromones by a functional VNO (Beckman, 2002; Keverne, 2002). Therefore, to directly assess the contribution of the VNO to sex discrimination, we subjected male mice to either surgical VNO removal (VNOx) or sham surgery (VNOi). The ability of such males to discriminate urinary odorants from male and female conspecifics or from male mice in different endocrine states was then compared in habituation-dishabituation tests. We also assessed subjects' preference to investigate male versus estrous female urinary odorants when presented simultaneously. One of the most dramatic phenotypes of TRP2 $2^{-1-}$ male mice was their display of high levels of mounting behavior instead of aggression toward other male conspecifics, which led to the conclusion that normal VNO function is needed for sex discrimination (Leypold et al., 2002; Stowers et al., 2002). Therefore, we also compared the mounting behavior directed by sexually experienced VNOi and VNOx males toward an estrous female as opposed to a castrated, urine-swabbed male presented simultaneously. 


\section{Materials and Methods}

Subjects. Fifty-eight male and 15 female C57BL/6 mice (Charles River Laboratories, Wilmington, MA) were purchased at 5-6 weeks of age and group housed under a reversed $12 \mathrm{hr}$ light/dark (L/D) photoperiod with food and water provided ad libitum. All of the procedures were approved by the Boston University Animal Care and Use Committee. All of the male subjects underwent bilateral castration via a single midline incision and at the same time received a subcutaneous implant of a SILASTIC capsule containing crystalline testosterone (length, $1.5 \mathrm{~cm} ; 0.04$ inch inner diameter $\times 0.085$ inch outer diameter) behind the neck (Pankevich et al., 2003). A minimum of $4 \mathrm{~d}$ later, animals underwent either bilateral removal of the vomeronasal organ or sham surgery. Removal of the VNO was accomplished via a midline incision in the roof of the mouth under ketamine $(120 \mathrm{mg} / \mathrm{kg})$ and xylazine $(12 \mathrm{mg} / \mathrm{kg})$ anesthesia. Briefly, animals were placed supine in a head holder, and the lower jaw was gently opened. A midline incision was made in the soft palette extending rostrally from behind the first palatal ridge to the incisors, and the underlying bone was exposed by blunt dissection. In VNOi animals, the incision was closed with absorbable sutures. For VNOx males, the rostral end of the VNO was exposed by drilling, the caudal end of the vomer bone was cut, and the VNO was removed bilaterally with a gentle twisting motion. Gel foam was packed into the cavity, and the incision was closed. Bleeding was controlled using a blunted 18 gauge needle attached to a vacuum. Animals were carefully monitored after surgery for bleeding and/or breathing difficulties and were allowed 1 week to recover before the onset of behavioral testing.

Behavioral tests. Urine was collected from five adult, gonadally intact C57BL/ 6 males, from five adult, C57BL/ 6 males that had been castrated 2 weeks before initial collection, and from 10 adult, ovariectomized C57BL/ 6 females both before and after they received estradiol benzoate (20 $\mu \mathrm{g}, 48$ and $24 \mathrm{hr}$ before collection) and progesterone (500 $\mu \mathrm{g}, 4 \mathrm{hr}$ before collection). Mice were held by the scruff of the neck, and urine was collected into tubes using a funnel. Urine from mice of the same sexendocrine state was pooled, thoroughly mixed, and stored in $30 \mu \mathrm{l}$ aliquots at $-80^{\circ} \mathrm{C}$. Before testing each day, aliquots of urine were thawed and used.

The ability of male mice to detect volatile as well as volatile and nonvolatile urinary odors was assessed separately using home-cage habituation-dishabituation tests (Baum and Keverne, 2002). Animals were housed alone in plastic cages measuring $29 \times 18 \times 13 \mathrm{~cm}$, and the bedding was not changed for a minimum of $48 \mathrm{hr}$ before any behavioral test. All of the behavioral tests were performed in the subjects' home cages during the dark phase of the $12 \mathrm{hr}$ L/D cycle using red light illumination. The experimenter was unaware of the surgical status of the subjects as well as the identity of the stimuli being presented in all of these tests. To assess the subjects' ability to discriminate pairs of volatile urinary odorants from each other, $10 \mu \mathrm{l}$ of urine from a particular source was pipetted onto a piece of filter paper that was taped to a plastic weigh boat. The cage top with food and water was removed and replaced with a clean top with a wire mesh barrier placed against the grid bars of the empty food hopper. A plastic weigh boat with the attached filter paper was placed behind the wire mesh so that only volatile odorants were available at body level, with subjects having no physical access to the stimulus. Subjects received three 2 min presentations of deionized water at $1 \mathrm{~min}$ intervals, followed by three $2 \mathrm{~min}$ presentations of one urinary odor and finally by three 2 min presentations of a second urinary odor. Using a Psion (London, UK) handheld computer and Noldus (Wageningen, The Netherlands) software, an observer recorded the number of seconds during each 2 min stimulus presentation in which a subject placed its nose against the grid bars opposite the filter paper containing the stimulus. In separate tests, subjects' ability to detect a combination of volatile and nonvolatile urinary cues was assessed by lowering weigh boats containing odorant stimuli via a fine wire along a wall inside the home cage. A record was kept of the time during each 2 min stimulus presentation in which subjects made direct nasal contact with the filter paper containing different odorant stimuli. For each pair of odorants presented either outside or inside the home cage, one-half of the subjects in the VNOi and VNOx groups were presented with the first urinary stimulus followed by the second odor, and the other half were presented with the same urinary odors but in the reverse order. Subsequently, on a second day of testing, all of the subjects were reexposed to the same odors but in the opposite order from their first day of exposure. The data from the $2 \mathrm{~d}$ of testing were then combined for each subject, whereupon within groups, two-tailed Wilcoxon tests were used to compare (1) the difference between the number of seconds that subjects spent investigating the third water stimulus versus the first urinary odorant presented and (2) the difference between the number of seconds spent investigating the third presentation of the first urinary stimulus versus the first presentation of the second urinary stimulus. Nonparametric Wilcoxon tests were used because of the relatively large number of "0" values obtained during the third presentation of either water or urinary odorants. Finally, subjects' preference to investigate one of a pair of odorants was assessed in separate 5 min tests, during which two odorants were presented simultaneously on pieces of filter paper attached to weigh boats that were placed side-by-side either outside or inside the home cage. Two-tailed Student's $t$ tests for paired samples were used to compare the times that subjects in each group spent investigating these respective odors.

Males' mating performance was observed twice for $4 \mathrm{hr}$ during the dark phase of the L/D cycle in the home cage after the addition of a hormone-primed, sexually receptive female. In studies using C57BL/6 mice, tests of this duration maximized the likelihood that ejaculation would be observed in the male (Baum et al., 1994). An observer recorded the incidence of anal-genital investigation, mounts with and without intromission, and ejaculation. After each observation session, subjects were left together for another $16 \mathrm{hr}$. For those few males in which the behavior was not observed during the formal $4 \mathrm{hr}$ mating test, the presence of a vaginal plug in the stimulus female the next day was taken as evidence of ejaculation. Additional tests were then given to assess subjects' preferred mounting partner, as described previously (Stowers et al., 2002). Both a hormone-primed, receptive female and a castrated male with intact male urine swabbed on the back were placed simultaneously in the home cage for $20 \mathrm{~min}$. These tests were videotaped and then later analyzed to determine (1) the percentage of mounts (with or without intromission) that each subject directed toward either the female or the male stimulus and (2) the total number of mounts displayed toward either stimulus. For each consecutive test, only data from males that showed more than two mounts toward either stimulus were included.

Experiment 1: sequence of tests. One week after VNO surgery, sexually naive male subjects were given habituation-dishabituation tests to assess their ability to discriminate intact male from estrous female urinary odorants. These urinary stimuli were presented in both sequences outside the home cage over $2 \mathrm{~d}$, followed by 2 more days of testing during which the same urinary stimuli were presented inside the home cage. Subsequently, subjects received 5 min simultaneous presentations of intact male and estrous female urine outside the home cage, followed on a subsequent day by simultaneous presentations inside the home cage. Subjects were then killed by transcardial perfusion with $0.1 \mathrm{M}$ PBS, $\mathrm{pH}$ 7.4 , followed by $4 \%$ paraformaldehyde in $0.1 \mathrm{~m}$ PBS. Olfactory bulbs were removed, postfixed for $4 \mathrm{hr}$ in $4 \%$ paraformaldehyde, and then placed in $30 \%$ sucrose $-0.1 \mathrm{M} \mathrm{PBS}$ for $4 \mathrm{~d}$ at $4^{\circ} \mathrm{C}$. Olfactory bulbs were then frozen in OCT (Tissue-Tek; Miles, Elkhart, IN) and stored at $-80^{\circ} \mathrm{C}$ until sectioning. Thirty micrometer sagittal sections were cut using a microtome with a freezing stage, and every other section was processed for soybean agglutinin conjugated with horseradish peroxidase (SBA-HRP) staining to ensure successful VNO removal (Wysocki and Wysocki, 1995). Although binding of SBA is selective for the accessory olfactory nerve and accessory olfactory bulb (AOB) glomeruli in the mouse (Key and Giorgi, 1986), the entire olfactory bulb was inspected to verify that no ectopic innervation from remnant VNO epithelium was present. There was never any SBA-HRP staining observed outside of the AOB. Only males in which SBA-HRP staining of the AOB was totally absent in both hemispheres were retained in the VNOx group (Fig. 1). For three mice in which VNO surgery was performed, although the glomerular layer was thinner than in control subjects, there was SBA-HRP staining in every section of both AOBs for two mice and in all but one of the sections from the right olfactory bulb of the third subject. Data from these mice were not used. 


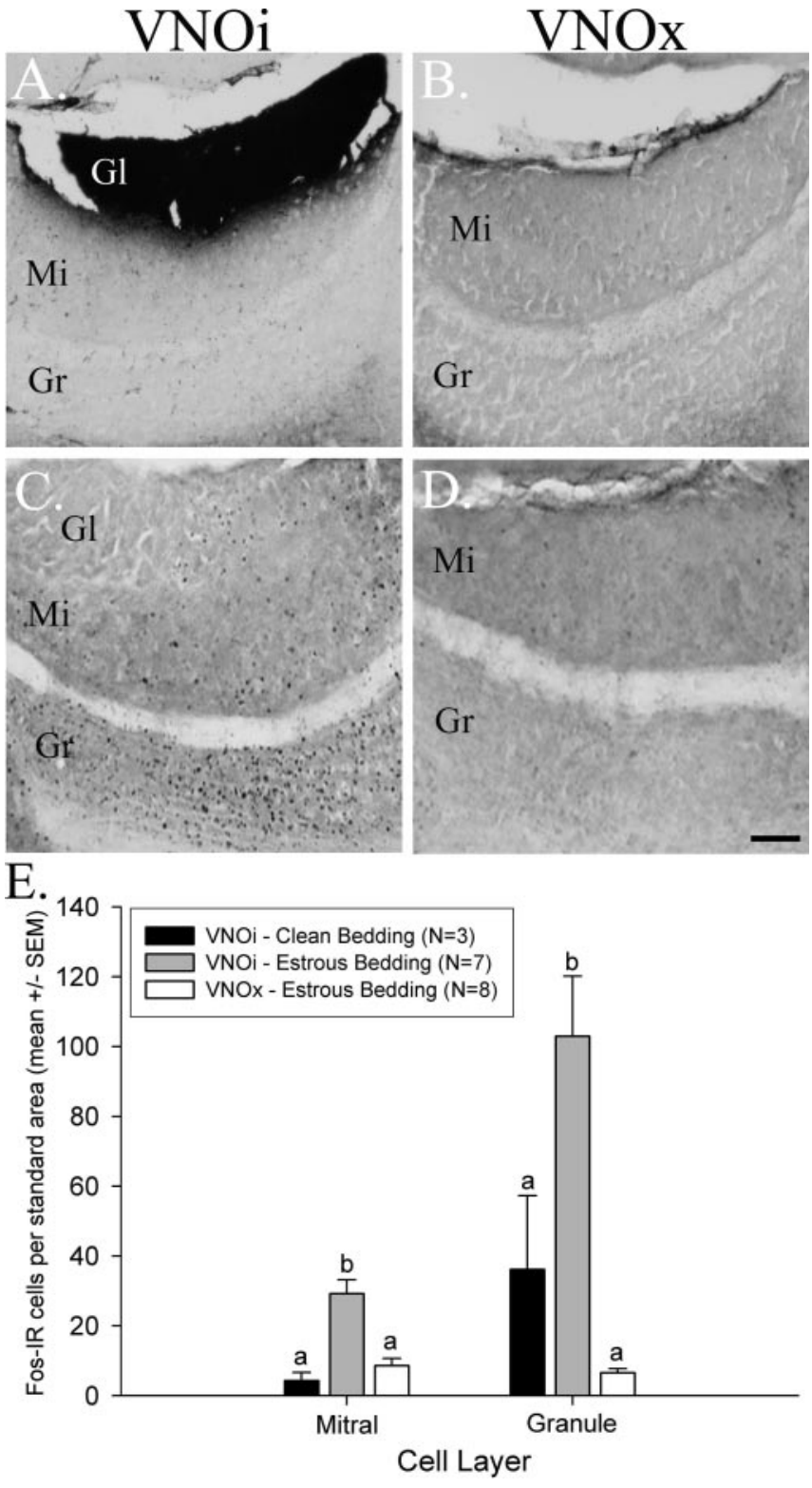

Figure 1. Representative photomicrographs show sagittal sections through the $A O B$ of male mice that had received either a sham removal of the vomeronasal organ or a bilateral surgical removal of the vomeronasal organ. $A, B$, Brain sections were stained with SBA-HRP.C, D, Brain sections immunolabeled for Fos protein are shown from VNOi and VNOx males exposed for 90 min to soiled bedding from estrous females before being killed. E, Mean \pm SEM counts of Fos-IR cells in AOB layers are compared from VNOi and VNOx males exposed to soiled bedding, as well as from VNOi males exposed to clean bedding. Induction of Fos in mitral and granule cell layers was significantly higher in VNOi males exposed to soiled bedding compared with the other groups. For each cell layer, means with different letters (a, b) are significantly different (Fisher's least significant difference tests, $p<0.01$ ). The absence of SBA-HRP staining in the glomerular layer $(B)$ and the background levels of Fos-IR cells in the AOB mitral and granule cell layers $(D)$ were taken as evidence of successful VNO removal. Gl, Glomerular cell layer; Mi, mitral cell layer; $\mathrm{Gr}$, granule cell layer. Scale bar, $100 \mu \mathrm{m}$.

Experiment 2: sequence of tests. Beginning 1 week after VNO surgery, a new group of males was given two mating tests with estrous females, conducted $5 \mathrm{~d}$ apart, after which the behavioral data from these two tests were combined. Subjects then received five tests of mounting partner preference at 5-6 dintervals. Finally, habituation-dishabituation tests of subjects' ability to distinguish volatile and nonvolatile components of two different pairs of odorants (ovariectomized vs estrous female; gonadally intact vs castrated male) were given. The presentation of these different pairs of urinary stimuli was always preceded by three consecutive 2 min presentations of deionized water. In this experiment, the odor stimuli were presented only inside the home cage because we reasoned that differences between VNOi and VNOx subjects would be most likely to occur in these circumstances. For each pair of odors, habituation-dishabituation tests were given on 2 separate days (reverse sequences of urinary odor presentation on each day), followed by a $5 \mathrm{~min}$ test of odor preference (simultaneous presentation of odors side-by-side inside the home cage) on a subsequent day. We also assessed subjects' preference to investigate urinary odors from estrous females versus gonadally intact males in a single 5 min test given after the completion of all of the other behavioral tests.

Subsequently, all of the subjects except for three VNOi males were exposed for 90 min to soiled bedding from estrous female mice, a stimulus that includes volatile and nonvolatile chemical signals found in bodily emissions. This stimulus has been found previously to induce the immediate early gene $c$-fos in the AOB granule and mitral cell layers (Halem et al., 2001). Three VNOi males were exposed to clean bedding as a control. Mice were then killed and perfused with $4 \%$ paraformaldehyde, and brains were collected as described in experiment 1 . Sagittal sections of the AOB were processed for either SBA-HRP staining or Fos immunoreactivity. Data from three subjects in experiment 1 were excluded based on the analysis of SBA-HRP staining in every other section through the AOB. To provide additional assurance that removal of the VNO was anatomically complete, every section from the left olfactory bulb in the experiment 2 subjects was processed and inspected for SBAHRP staining. Every other section from the right olfactory bulb was also stained with SBA-HRP, and the alternate sections were processed for Fos immunocytochemistry as described previously (Halem et al., 2001). For quantification of Fos induction, two anatomically matched sections were selected from each subject, and Fos-immunoreactive (IR) cells were identified and counted separately for the $\mathrm{AOB}$ mitral and granule cell layers.

After examination of the SBA-HRP-labeled sections, two males were rejected: in one of the males, $\mathrm{AOB}$ labeling was seen in two sections, whereas in the right olfactory bulb of this male and both olfactory bulbs of the second male, the AOB glomerular layer was labeled in every section examined. Thus, in every subject rejected from experiment 1 or 2, labeled processes extended through at least two but typically most or all sections of the AOB, so it is unlikely that any SBA-HRP-labeled fibers were missed in olfactory bulbs of subjects in which only every other section was stained.

\section{Results \\ Experiment 1}

VNOi and VNOx male mice were able to both detect and discriminate between volatile urinary odors from intact males and estrous females (Fig. 2A). Thus, significant increases in investigation times (dishabituation) were seen in both groups of males during initial presentation of a urinary stimulus after the third presentation of water. Also, both groups showed significant increases in investigation times during the initial switch from male to estrous female urinary odors (or vice versa) presented outside the home cage. Males spent progressively less time investigating each urinary stimulus after its first presentation (habituation). When the same stimuli were moved inside the subjects' home cage, allowing access to both volatile and nonvolatile urinary odorants, both VNOi and VNOx males were again able to discriminate between odorants emitted from male versus estrous female urine (Fig. 2B). Subjects in the two groups investigated male and estrous female volatile urinary odors equally when they were presented simultaneously for 5 min outside the subjects' home cages (Fig. 2C, left). However, when these stimuli were moved inside subjects' home cages, thereby providing access to both volatile and nonvolatile urinary odorants, VNOi males spent significantly more time investigating the estrous female as opposed to intact male urine $\left(t_{(4)}=2.483 ; p<0.05\right)$, whereas 


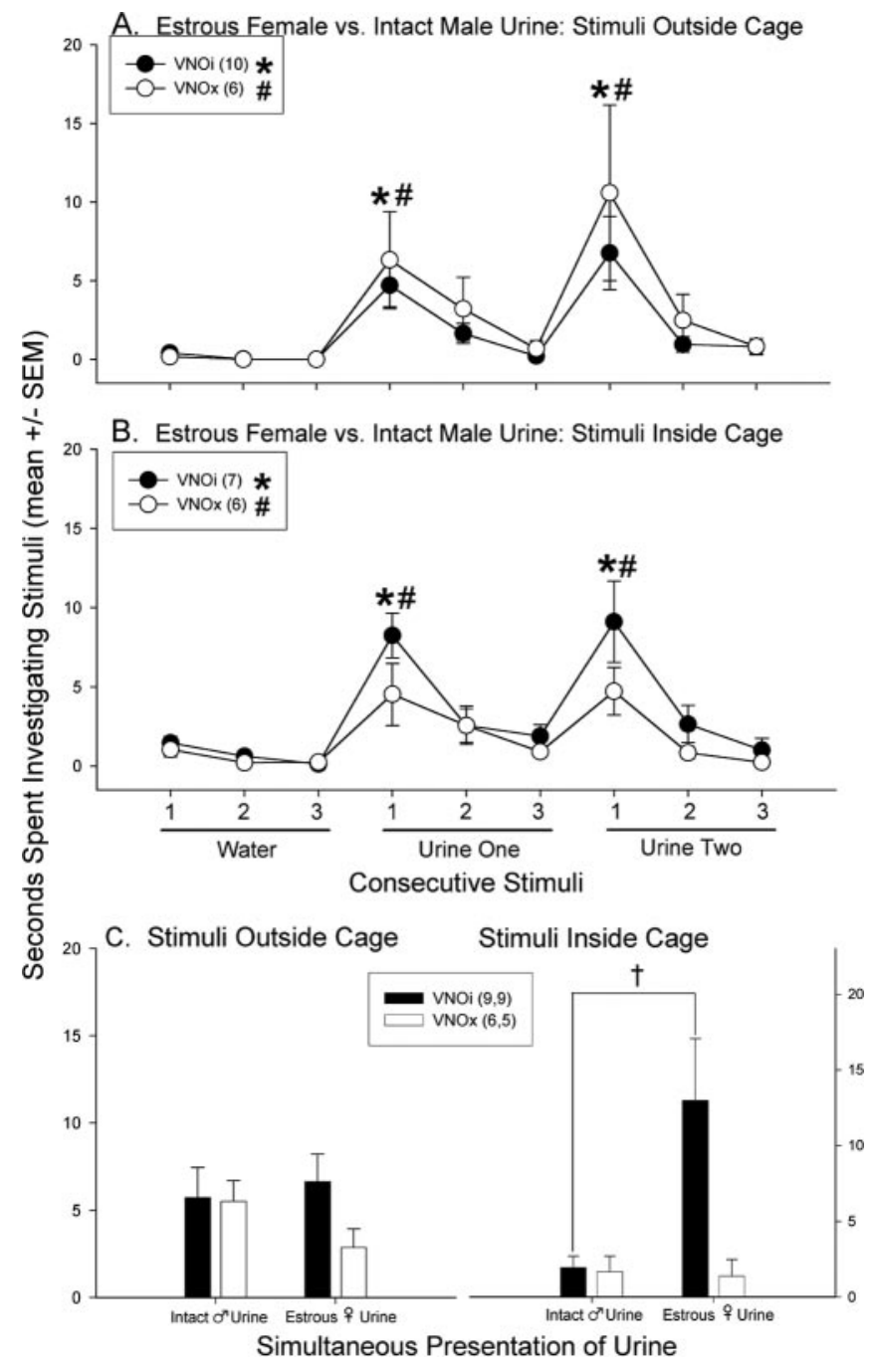

Figure 2. Effect of bilateral removal of the vomeronasal organ or sham operation on the ability of male mice to discriminate volatile urinary odors from gonadally intact male versus estrous female mice when urine was presented outside the home cage $(A)$ or to discriminate volatile and nonvolatile components of these two kinds of urine when presented inside the home cage $(B)$. In both situations, subjects received a habituation- dishabituation test during which investigation times were recorded in nine total trials that consisted of three presentations of water, followed by three presentations of one of the urinary odors, followed by three presentations of the second urinary odor. The test was repeated for each subject on a separate day but with the order of the odors reversed. Data from the two tests were combined for each subject, and the mean \pm SEM seconds that VNOi and VNOx males spent investigating stimuli is shown for each trial, with Urine One representing the first odor presented and Urine Two representing the second odor presented. ${ }^{*} \# p<0.05$, two-tailed Wilcoxon test comparisons with the third presentation of the previous stimulus for the respective groups. The time that subjects spent investigating the same two of these urinary odors when presented simultaneously outside $\left(C\right.$, left) or inside $\left(C\right.$, right) the home cage is also shown. ${ }^{\dagger} p<0.05$, two-tailed paired Student's $t$ test. The number of subjects in each group that was tested under each condition is shown in parentheses.

VNOx males investigated the two odor stimuli for equal times (Fig. 2C, right).

\section{Experiment 2}

Over two $4 \mathrm{hr}$ mating sessions, VNOi and VNOx male mice showed high levels of sexual behavior, including ejaculation; there were no significant group differences in any of the parameters of sexual behavior recorded (Table 1).

When VNOi and VNOx male mice were tested over a 1 month
Table 1. Effect of vomeronasal organ removal on sexual behavior of sexually naive male mice

\begin{tabular}{lcc}
\hline Behavior & VNOi $(n=11)$ & VNOx $(n=9)$ \\
\hline Anogenital investigation & & \\
$\quad$ Percentage showing & 100 & 100 \\
$\quad$ Latency (min) & $26.5 \pm 10.7$ & $15.5 \pm 12.6$ \\
$\quad$ Number per test & $4.8 \pm 0.8$ & $4.5 \pm 1.3$ \\
Mounts (with or without intromission) & 100 & 100 \\
$\quad$ Percentage showing & $23.3 \pm 16.8$ & $15.3 \pm 6.6$ \\
$\quad$ Latency (min) & $35.5 \pm 4.6$ & $28.0 \pm 7.5$ \\
$\quad$ Number per test & $1.5 \pm 0.3$ & $0.9 \pm 0.3$ \\
$\quad$ Mounts per minute & 100 & \\
Mounts (with intromission) & $27.1 \pm 14$ & 100 \\
$\quad$ Percentage showing & $20.8 \pm 2.9$ & $21.1 \pm 9.6$ \\
$\quad$ Latency (min) & $1.0 \pm 0.2$ & $0.6 \pm 0.0$ \\
$\quad$ Number per test & $3.1 \pm 1.0$ & $3.4 \pm 0.9$ \\
$\quad$ Mounts (with intromission) per minute & $27.0 \pm 6.4$ & $26.6 \pm 8.6$ \\
Interintromission interval (min) & & \\
Percentage of mounts leading to intromission & 91 & 100 \\
Ejaculations & & \\
$\quad$ Percentage showing & $29.4 \pm 4.3$ & \\
$\quad$ Latency (min) & &
\end{tabular}

period with an estrous female and a castrated male (with intact male urine swabbed on the back) presented simultaneously, both groups directed an equivalent $(60-70 \%)$ percentage of their mounts toward the estrous female, with the remaining mounts being directed toward the castrated male (Fig. 3, top). Likewise, VNOi and VNOx males showed a similar total number of mounts per test (directed toward either stimulus animal) over the five tests (Fig. 3, bottom).

VNOi and VNOx male subjects showed similar, significant dishabituation responses when urinary odors from estrous females versus ovariectomized females and from gonadally intact versus castrated males were presented inside the home cage (Fig. $4 A, B)$. When each of these pairs of odorants was presented simultaneously inside the home cage for $5 \mathrm{~min}$, VNOi and VNOx animals investigated urine spots from both the estrous and ovariectomized females for equal times, whereas both VNOi $\left(t_{(8)}=\right.$ $-4.29 ; p<0.01)$ and VNOx $\left(t_{(4)}=-4.40 ; p<0.05\right)$ males showed a significant preference to investigate urine from castrated as opposed to gonadally intact males (data not shown). As in experiment 1 , when presented simultaneously inside the home cage, VNOi subjects showed a significant preference to investigate estrous female over intact male urine $\left(t_{(10)}=2.28 ; p<0.05\right)$, whereas VNOx males investigated these stimuli for an equal amount of time (Fig. 4C).

To determine the functional efficacy of VNO removal, alternate sections of the left olfactory bulb were processed for Fos immunocytochemistry, and the mean number of Fos-IR cells occurring in the $\mathrm{AOB}$ of each group were compared by one-way ANOVA and Fisher's least significant difference post hoc tests. Of the subjects exposed to soiled estrous bedding, VNOx males exhibited significantly fewer Fos-IR cells than VNOi males in both the mitral $\left(F_{(2,15)}=18.8 ; p<0.0001\right)$ and granule $\left(F_{(2,15)}=17.5\right.$; $p<0.0001)$ cell layers. Moreover, the level of Fos activation in the AOB of VNOx males exposed to soiled estrous bedding was no different from that seen in VNOi males that had been exposed to clean bedding. These results provide additional confirmation that no pheromonal cues are processed by the accessory olfactory system after surgical removal of the VNO. 

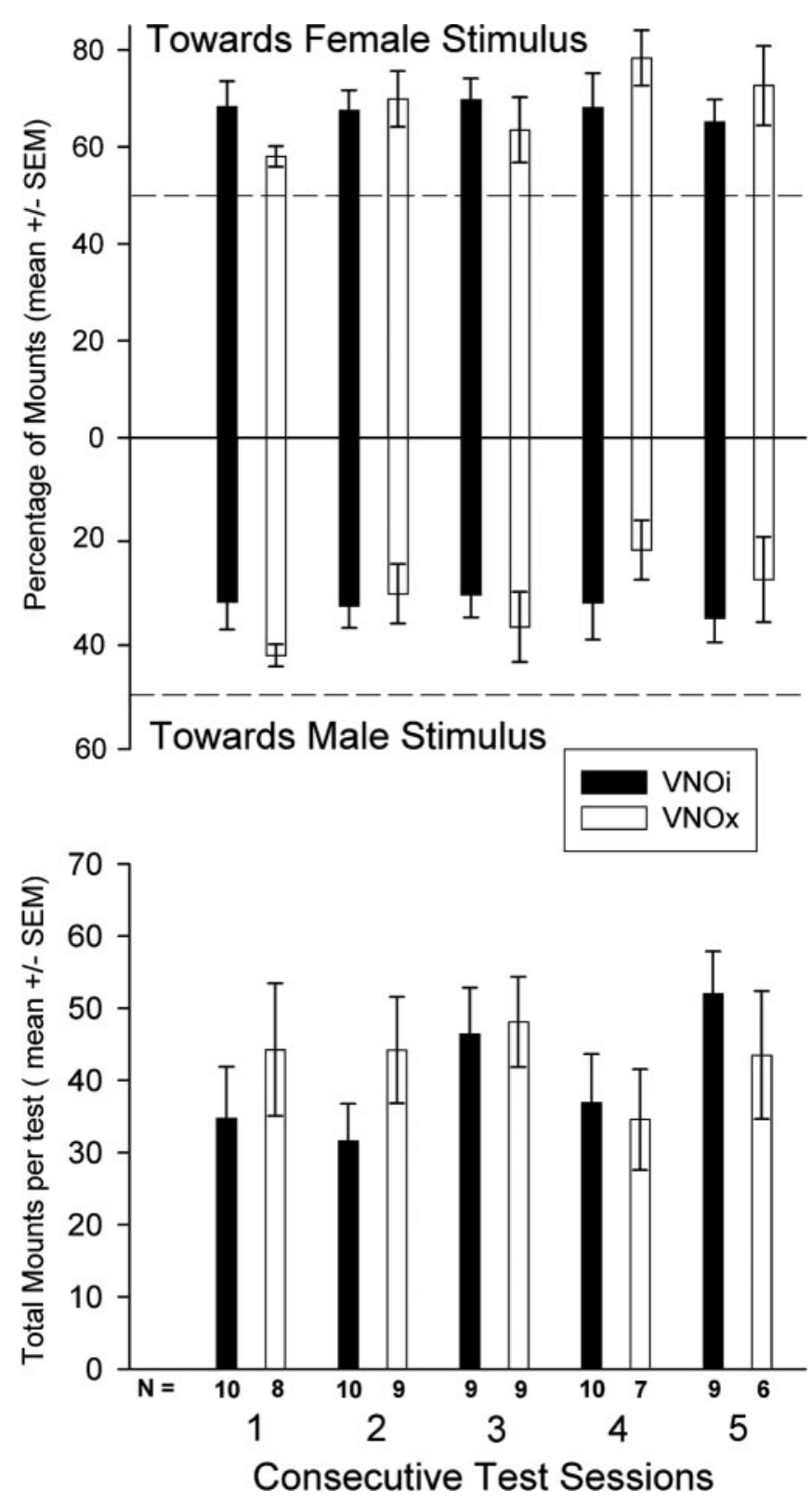

Figure 3. Effect of bilateral removal of the vomeronasal organ or sham operation in male mice on the percentage of mounts directed toward an estrous female versus a castrated male stimulus with intact male urine swabbed on the back when presented simultaneously for 20 min in the subjects' home cages (top). The dotted horizontal lines show the $50 \%$ preference for each type of stimulus. The total number of mounts per test displayed by VNOi and VNOx subjects toward both stimulus mice is shown at the bottom. Results for each test are based only on male subjects that displayed more than two mounts toward either stimulus animal (the number of subjects is given across the $x$-axis).

\section{Discussion}

After VNO removal, male mice were still able to reliably distinguish between urinary odors from males and estrous females and from mice of both sexes that were in different endocrine states. These results are in agreement with studies in which VNO removal failed to disrupt the ability of male guinea pigs (Beauchamp et al., 1982) or female hamsters (Petrulis et al., 1999) to discriminate between odorants emitted from males versus females. Moreover, a consistent finding of the present study seen in sexually naive as well as experienced male mice was that VNO removal significantly attenuated subjects' motivation to spend

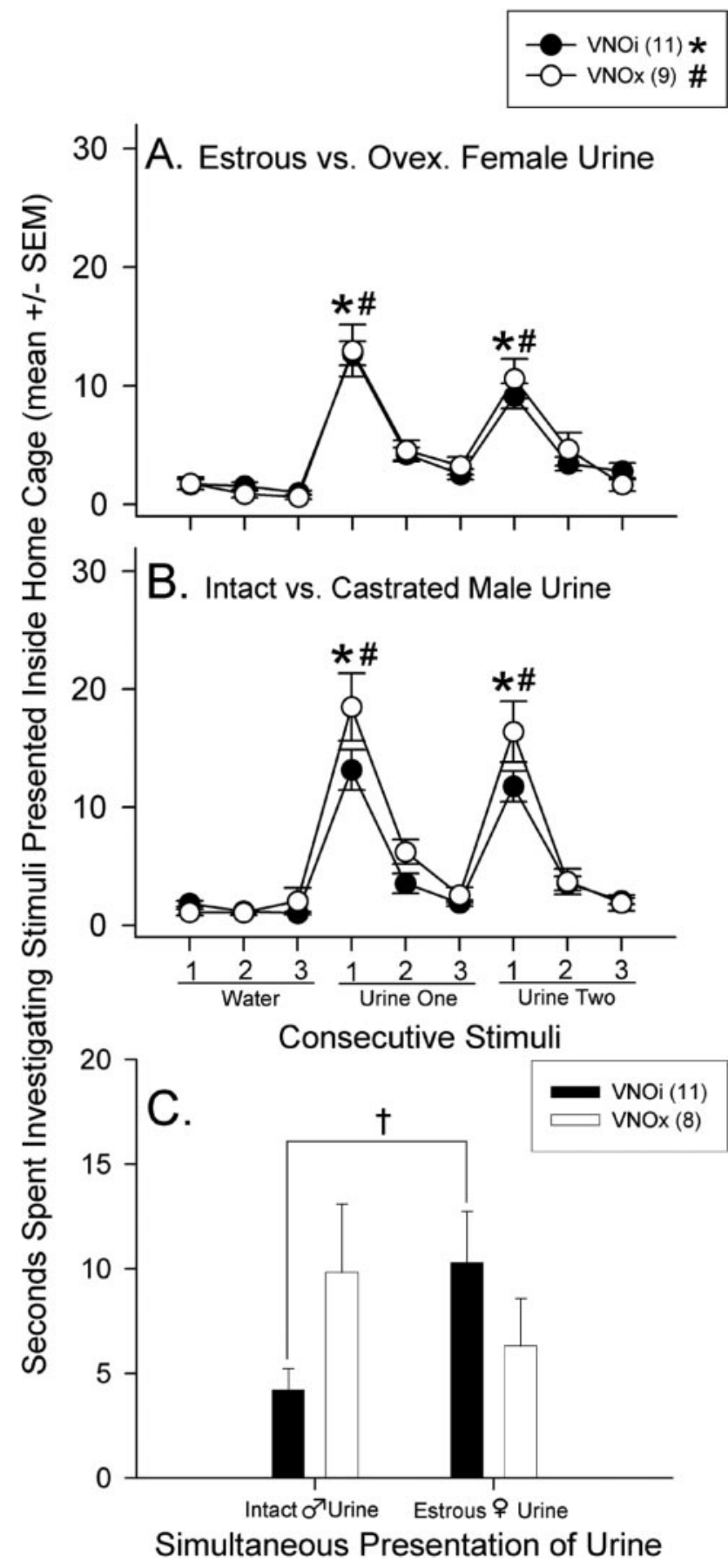

Figure 4. Effect of bilateral removal of the vomeronasal organ or sham operation on the ability of male mice to discriminate volatile and nonvolatile urinary odors (presented inside the home cage) from estrous versus ovariectomized (0vex.) females $(A)$ or from gonadally intact versus castrated males $(B)$. Subjects were given habituation- dishabituation tests as described in Figure 2, and the data are similarly presented as the mean \pm SEM seconds that VNOi and VNOx males spent investigating stimuli on each trial. ${ }^{*}, p<0.05$, two-tailed Wilcoxon test comparisons with the third presentation of the previous stimulus for the respective groups. The time that subjects spent investigating gonadally intact male versus estrous female urine when presented simultaneously inside the home cage is also shown ( ()$^{\dagger}{ }^{\dagger} p<0.05$, two-tailed paired Student's $t$ test. The number of subjects in each group that was tested under each condition is shown in parentheses.

time in nasal contact with a spot of estrous female as opposed to male urine. The results of habituation-dishabituation tests using the same urinary stimuli strongly suggest that this difference reflected a motivational deficit of VNOx males as opposed to an 
inability to discriminate between the two stimuli. A similar conclusion was proposed by Beauchamp et al. (1982), who observed that VNO removal reduced the preference of male guinea pigs to spend time investigating urine from females without disrupting subjects' ability to discriminate the two types of odorants. These results corroborate the early view (Powers et al., 1979; O'Connell and Meredith, 1984) that the main olfactory system is used to identify and localize conspecifics according to their sex and endocrine status. After contact, however, nonvolatile components of urinary or other body odorants may reach VNO receptors and lead to their activation, with behavioral and neuroendocrine consequences.

All of these findings contradict the recent conclusion (Leypold et al., 2002; Stowers et al., 2002) that VNO function underlies the ability of mice to discriminate the sex of conspecifics. TRP2 $2^{-1-}$ male mice displayed mounting behavior instead of aggression toward a passive male intruder (Leypold et al., 2002; Stowers et al., 2002), and they also displayed equal levels of mounting directed toward a castrated male and an estrous female presented simultaneously (Stowers et al., 2002). In the study by Stowers et al. (2002), no wild-type control males were tested; thus, it is impossible to say whether their mounting preferences would have differed from $T R P 2^{-/-}$males. In the study by Leypold et al. (2002), sexually experienced TRP2 $2^{-1-}$ and wild-type control males preferred to mount a nonestrous female over a male, although the preference was considerably stronger in wild-type males. This difference may have been less evident if estrous instead of diestrous females had been paired with castrated males during these tests. In the present study, VNOi and VNOx C57BL/6 male mice, the same background strain as that used by Leypold et al. (2002) and Stowers et al. (2002), both directed $>50 \%$ of their mounts toward an estrous female, whereas both groups directed an equivalent, minority of mounts toward a castrated male. This bisexual mounting behavior was seen in both groups of males when tested five times over a 1 month period, suggesting that it reflects a stable characteristic of sexual motivation in this mouse strain regardless of whether or not the VNO is functional. A similar conclusion was reached by Del Punta et al. (2002), who consistently observed equivalent, albeit low, levels of mounting directed toward intruder male mice in male subjects lacking a cluster of $16 \mathrm{~V} 1 \mathrm{r} \mathrm{VNO}$ receptor genes, as well as in wild-type controls. Likewise, males with a mutation of the $G_{\alpha 2 i}$ gene resembled wild-type controls in their preference to mount an estrous female over a castrated male (Norlin et al., 2003).

Although it has been claimed (Trinh and Storm, 2003) that volatile constituents of urine may directly activate VNO sensory neurons, AOB mitral cells were only activated when male mice came into direct physical contact with the head or anogenital regions of conspecifics, and exposure to volatile urinary odors failed to activate these neurons (Luo et al., 2003). Thus, the strongest available evidence suggests that only nonvolatile constituents of urine and/or other body odorants activated VNO sensory neurons. Our results suggest that volatile components of urine alone are able to inform male mice of the sex of conspecifics. Thus, in experiment 1, both VNOi and VNOx males showed significant dishabituation responses to urinary odors of male versus estrous female mice when these stimuli were presented outside the home cage. The ability of VNOx males to perform this task implies that it was subserved by the main olfactory epithelium (MOE) instead of the VNO. This conclusion is supported further by a study ( $\mathrm{Ma}$ et al., 2002) in which MOE neurons were selectively destroyed by intranares administration of a toxin to transgenic female mice expressing a bacterial nitroreductase enzyme whose expression was driven by the olfactory marker protein promoter. These transgenic, drug-treated female mice failed to show dishabituation responses to male urinary odors, regardless of whether the stimuli were presented outside or inside the home cage, but showed a robust pregnancy block after exposure to soiled bedding from a strange male $2 \mathrm{~d}$ after mating with a stud male of a different strain. This suggests that the VNO was functional in these females, yet they were unable to localize or discriminate urinary odors from opposite-sex conspecifics. Other results (Schaefer et al., 2001, 2002) showed that glomeruli in the main olfactory bulb were activated in female mice after exposure to volatile urinary odors from males.

A change in motivational status may account for the reduction in aggression displayed toward a male intruder (Clancy et al., 1984), as well as reductions in ultrasonic vocalizations emitted toward estrous female conspecifics (Wysocki et al., 1982) observed previously in VNOx male mice. In each instance, there was no evidence that VNOx males were unable to use olfactory cues to discriminate the sex of stimulus animals. Instead, their display of aggression and ultrasonic vocalizations in response to male and female stimuli, respectively, was reduced. In contrast, whereas $\mathrm{TRP}^{-/-}$male mice (like VNOx males) showed no aggression toward male intruders, unlike VNOx males they showed equivalent levels of ultrasonic vocalization in the presence of a female or a castrated male (Stowers et al., 2002). In our study, a complete elimination of VNO signaling was confirmed both anatomically (absence of SBA-HRP staining in the AOB) and functionally (absence of odor-induced Fos immunoreactivity in the AOB). Perhaps the differences between VNOx and TRP2 $2^{-/-}$males reflects the difference between a total elimination of VNO inputs to the AOB seen after VNOx as opposed to a distortion of synaptic inputs to the AOB from the VNO that could occur in TRP2 $2^{-/-}$ mice or in mice lacking a set of VNO receptor genes (Del Punta et al., 2002; Leypold et al., 2002).

It is noteworthy that the rates of mounting and intromitting, which is critical for the induction of a progestational state in pregnant females (Diamond, 1970), and the timing of ejaculation were equivalent in sexually naive VNOi and VNOx males. This outcome differs dramatically from results in hamsters (Meredith, 1986) in which VNO destruction disrupted mating performance in sexually naive as opposed to experienced males. At first blush, the present result resembles those obtained for TRP2 ${ }^{-1-}$ male mice (Leypold et al., 2002; Stowers et al., 2002) whose mating performance was equivalent to wild-type controls. Those studies used 15-30 min tests in which males in both groups ejaculated only infrequently. Therefore, the reported absence of any difference in mating performance between those genotypes is not definitive. The same limitation applies to a previous study (Wysocki and Lepri, 1991) that observed similar levels of "total sexual behavior" scores in sexually experienced VNOi and VNOx male mice during 20 min tests given over a 3 week period. In subsequent tests, sexual behavior scores of VNOx males diminished significantly, perhaps, as argued by the authors, because these males were deprived of the reinforcing influence of VNO inputs to the brain otherwise derived from mating. In another study (Clancy et al., 1984), VNO removal reduced mount and intromission frequency in male mice without affecting ejaculation. Clancy et al. (1984) tested mice twice, once for $15 \mathrm{~min}$ and again for $1 \mathrm{hr}$, during the light phase of the light/dark cycle. Our use of two $4 \mathrm{hr}$ tests given during the dark (active) phase of the light/ dark cycle provided the opportunity to assess mating performance over extended periods during the portion of the cycle in which mice normally mate. This procedural difference may have 
led to the observed similarity in the mating performance of $\mathrm{VNO}$ and VNOx males.

In the present experiments, $\mathrm{VNO}$ removal failed to affect subjects' ability to discriminate sex or endocrine status based on olfactory cues, whereas it disrupted males' preference to investigate estrous female versus male urine presented inside the home cage. This latter effect occurred before (experiment 1) and after (experiment 2) the mating experience, suggesting that nonvolatile olfactory cues from estrous females, which are detected by the $\mathrm{VNO}$, may be intrinsically attractive to males even before they are associated with mating. In more natural spatial and social environments than those typically used in laboratory studies, the attraction engendered by $\mathrm{VNO}$-mediated, olfactory inputs to the forebrain may prolong physical contact with estrous females, thereby contributing to reproductive success in male mice.

\section{References}

Baum MJ, Keverne EB (2002) Sex difference in attraction thresholds for volatile odors from male and estrous female mouse urine. Horm Behav 41:213-219.

Baum MJ, Brown JJ, Kica E, Rubin BS, Johnson RS, Papaioannou VE (1994) Effect of a null mutation of the c-fos proto-oncogene on sexual behavior of male mice. Biol Reprod 50:1040-1048.

Beauchamp GK, Martin IG, Wysocki CJ, Wellington JL (1982) Chemoinvestigatory and sexual behavior of male guinea pigs following vomeronasal organ removal. Physiol Behav 29:329-336.

Beckman M (2002) When in doubt, mice mate rather than hate. Science 295:782.

Clancy AN, Coquelin A, Macrides F, Gorski RA, Noble EP (1984) Sexual behavior and aggression in male mice: involvement of the vomeronasal system. J Neurosci 4:2222-2229.

Del Punta K, Leinders-Zufall T, Rodriguez I, Jukam D, Wysocki CJ, Ogawa S, Zufall F, Mombaerts P (2002) Deficient pheromone responses in mice lacking a cluster of vomeronasal receptor genes. Nature 419:70-74.

Diamond M (1970) Intromission pattern and species vaginal code in relation to induction of pseudopregnancy. Science 169:995-997.

Halem HA, Baum MJ, Cherry JA (2001) Sex difference and steroid modulation of pheromone-induced immediate early genes in the two zones of the mouse accessory olfactory system. J Neurosci 21:2474-2480.

Halpern M, Martinez-Marcos A (2003) Structure and function of the vomeronasal system: an update. Prog Neurobiol 70:245-318.

Keverne EB (2002) Pheromones, vomeronasal function, and genderspecific behavior. Cell 108:735-738.

Key B, Giorgi PP (1986) Soybean agglutinin binding to the olfactory systems of the rat and mouse. Neurosci Lett 69:131-136.

Leypold BG, Yu CR, Leinders-Zufall T, Kim MM, Zufall F, Axel R (2002)
Altered sexual and social behaviors in trp2 mutant mice. Proc Natl Acad Sci USA 99:6376-6381.

Luo M, Fee MS, Katz LC (2003) Encoding pheromonal signals in the accessory olfactory bulb of behaving mice. Science 299:1196-1201.

Ma D, Allen ND, Van Bergen YC, Jones CM, Baum MJ, Keverne EB, Brennan PA (2002) Selective ablation of olfactory receptor neurons without functional impairment of vomeronasal receptor neurons in OMP-ntr transgenic mice. Eur J Neurosci 16:2317-2323.

McCarthy MM, Auger AP (2002) He's a lover, not a fighter-smell, sex and civility. Trends Endocrinol Metab 13:183-184.

Meredith M (1986) Vomeronasal organ removal before sexual experience impairs male hamster mating behavior. Physiol Behav 36:737-743.

Norlin EM, Gussing F, Berghard A (2003) Vomeronasal phenotype and behavioral alterations in G alpha i2 mutant mice. Curr Biol 13:1214-1219.

O'Connell RJ, Meredith M (1984) Effects of volatile and nonvolatile chemical signals on male sex behaviors mediated by the main and accessory olfactory systems. Behav Neurosci 98:1083-1093.

Pankevich D, Baum MJ, Cherry JA (2003) Removal of the superior cervical ganglia fails to block Fos induction in the accessory olfactory system of male mice after exposure to female odors. Neurosci Lett 345:13-16.

Petrulis A, Peng M, Johnston RE (1999) Effects of vomeronasal organ removal on individual odor discrimination, sex-odor preference, and scent marking by female hamsters. Physiol Behav 66:73-83.

Powers JB, Fields RB, Winans SS (1979) Olfactory and vomeronasal system participation in male hamsters' attraction to female vaginal secretions. Physiol Behav 22:77-84.

Schaefer ML, Young DA, Restrepo D (2001) Olfactory fingerprints for major histocompatibility complex-determined body odors. J Neurosci 21:2481-2487.

Schaefer ML, Yamazaki K, Osada K, Restrepo D, Beauchamp GK (2002) Olfactory fingerprints for major histocompatibility complex-determined body odors II: relationship among odor maps, genetics, odor composition, and behavior. J Neurosci 22:9513-9521.

Stowers L, Holy TE, Meister M, Dulac C, Koentges G (2002) Loss of sex discrimination and male-male aggression in mice deficient for TRP2. Science 295:1493-1500.

Trinh K, Storm DR (2003) Vomeronasal organ detects odorants in absence of signaling through main olfactory epithelium. Nat Neurosci 6:519-525.

Wysocki CJ, Lepri JJ (1991) Consequences of removing the vomeronasal organ. J Steroid Biochem Mol Biol 39:661-669.

Wysocki CJ, Wysocki LM (1995) Surgical removal of the mammalian vomeronasal organ and its verification. In: Experimental cell biology of taste and olfaction (Speilman AI, Brand JG, eds), pp 49-57. New York: CRC.

Wysocki CJ, Nyby J, Whitney G, Beauchamp GK, Katz Y (1982) The vomeronasal organ: primary role in mouse chemosensory gender recognition. Physiol Behav 29:315-327.

Yang A, Walker N, Bronson R, Kaghad M, Oosterwegel M, Bonnin J, Vagner C, Bonnet H, Dikkes P, Sharpe A, McKeon F, Caput D (2000) p73deficient mice have neurological, pheromonal and inflammatory defects but lack spontaneous tumours. Nature 404:99-103. 